\title{
Biomarker Discovery via Optimal Bayesian Feature Filtering for Structured Multiclass Data
}

\author{
Ali Foroughi pour \\ Department of Electrical and Computer Engineering \\ The Ohio State University \\ Columbus, Ohio \\ foroughipour.1@osu.edu
}

\author{
Lori A. Dalton \\ Department of Electrical and Computer Engineering \\ The Ohio State University \\ Columbus, Ohio \\ dalton@ece.osu.edu
}

\begin{abstract}
Biomarker discovery aims to find a shortlist of high-profile biomarkers that can be further verified and utilized in downstream analysis. Many biomarkers exhibit structured multiclass behavior, where groups of interest may be clustered into a small number of patterns such that groups assigned the same pattern share a common governing distribution. While several algorithms are proposed for multiclass problems, to the best of our knowledge, none can take such constraints on the group-pattern assignment, or structure, as input, and output high-profile potential biomarkers as well as the structure they satisfy. While post analyses may be used to infer the structure, ignoring such information impedes feature selection to fully take advantage of experimental data. Recent work proposes a Bayesian framework for feature selection that places priors on feature-label distribution and label-conditioned feature distribution. Here we extend this framework for structured multiclass problems, solve the proposed model for the case of independent features, evaluate it in several synthetic simulations, apply it to two cancer datasets, and perform enrichment analysis. Many of the highly ranked genes and pathways are suggested to be affected in the cancer under study. We also find potentially new biomarkers. Not only do we detect biomarkers, but also make inferences about the underlying distributional connections across classes, which provide additional insight on cancer biology.
\end{abstract}

\section{CCS CONCEPTS}

- Mathematics of computing $\rightarrow$ Bayesian computation; • Theory of computation $\rightarrow$ Bayesian analysis; $\cdot$ Computing methodologies $\rightarrow$ Feature selection;

\section{KEYWORDS}

biomarker discovery, feature selection, Bayesian analysis, multiclass data, structured data

ACM Reference Format:

Ali Foroughi pour and Lori A. Dalton. 2018. Biomarker Discovery via Optimal Bayesian Feature Filtering for Structured Multiclass Data. In ACMBCB'18: 9th ACM International Conference on Bioinformatics, Computational

Permission to make digital or hard copies of all or part of this work for personal or classroom use is granted without fee provided that copies are not made or distributed for profit or commercial advantage and that copies bear this notice and the full citation on the first page. Copyrights for components of this work owned by others than ACM must be honored. Abstracting with credit is permitted. To copy otherwise, or republish to post on servers or to redistribute to lists, requires prior specific permission and/or a fee. Request permissions from permissions@acm.org.

ACM-BCB'18, August 29-September 1, 2018, Washington, DC, USA

(C) 2018 Association for Computing Machinery.

ACM ISBN 978-1-4503-5794-4/18/08 \&\$15.00

https://doi.org/10.1145/3233547.3233558
Biology and Health Informatics, August 29-September 1, 2018, Washington, DC, USA. ACM, New York, NY, USA, 10 pages. https://doi.org/10.1145/3233547. 3233558

\section{INTRODUCTION}

Biomarker discovery aims to find a shortlist of biomarkers across two or several groups, such as patients with different subtypes of cancer or patients at different stages of cancer. The goal is to identify potential biomarkers that are differentially expressed, i.e., have distributional differences among groups, and thus may be involved in the biological mechanisms of the disease under study. Verified biomarkers can be further utilized for various applications such as diagnosis, drug development, and personalized treatment [25]. While current high-throughput technologies provide a deluge of data per point, research is usually constrained to small samples, which impedes reliable biomarker discovery [32, 48, 49].

Many biomarkers exhibit structured multiclass behavior, where groups of interest may be clustered into a small number of patterns such that groups assigned the same pattern share a common governing distribution. In this work, we call a group-pattern assignment a structure. Furthermore, in many applications it is possible to take advantage of structure constraints to aid biomarker discovery. We provide 2 examples to illustrate. Consider a multiclass problem with 5 classes: healthy people, and cancer patients in stages $1,2,3$, and 4 . Figure 1(a) presents some of the possible structures. Rows represent different classes and columns represent marker families. A marker corresponding to the first column follows one distribution among healthy people and another distribution among all cancer patients, suggesting it might be involved in initiating cancer. Alternatively, a biomarker corresponding to column 2 follows the same distribution among healthy people and stage 1 cancer patients, and another distribution among stage 2, 3, and 4 cancer patients. Hence, such marker might be involved in cancer progression. A similar argument holds for markers in columns 3 and 4. An example of such markers is provided in sec. 4.1. Finally, column 5 depicts non-markers, i.e., features that have the same distribution among all classes. This example illustrates the importance of considering marker structures in biomarker discovery. Knowing the cancer stage at which a biomarker starts to behave differently provides invaluable insight on cancer biology. For instance, biomarkers affected in later stages may initiate late stage events such as metastasis.

As another example consider a 3 class problem: healthy people, subtype 1 cancer patients, and subtype 2 patients. Figure 1(b) presents some of the possible structures. Rows represent classes and columns represent marker families. A marker in column 1 follows one distribution among healthy people, and another distribution among cancer patients. This marker is affected in the same way 
in both cancer subtypes. A marker in column 2 follows one distribution among healthy people and subtype 1 cancer patients, and another distribution among subtype 2 patients, i.e., is only affected in subtype 2 . We find markers only affected in specific leukemia subtypes in sec. 4.2. Last column represents non-markers. The example illustrates how structures help study differences among cancer subtypes, which is useful in various applications such as developing treatment protocols that target specific cancer subtypes.

Various algorithms are proposed for biomarker discovery, of which many optimize over heuristic objectives related to selecting differentially expressed features. For example, t-test and ANalysis Of VAriance (ANOVA) detect differences in means, mutual information selects features that reduce uncertainty on class labels, and forward selection using Hilbert-Schmidt independence criterion (FOHSIC) [53] finds a set of features highly correlated with class labels. Wrapper methods assign a cost based on a classification error estimate to each feature set, but may report few features due to peaking phenomenon [49], the feature set minimizing the error estimate might not be close to the set with minimal Bayes error [48], and their performance degrades as number of classes increase [32]. None of these algorithms take a set of possible structures as input. Post analyses, such as pairwise analyses based on ANOVA, can be used to further study distributional similarities and differences, but they are not directly enforced at the selection stage. To the best of our knowledge, the proposed feature selection algorithms are the first methods that take structure constraints as input, and impose them at the feature selection stage. Note structural constraints on possible forms of distributional differences that a marker can follow is different than structural constraints on marker families. For instance, while group LASSO [55] assumes a known grouping of features where features in the same group are all markers or non-markers, it does not consider distributional constraints across classes. While group LASSO might declare a group of features are all markers, in the example of Fig. 1(a) it does not specify if they follow the marker structure of the first column, or the second.

Recent work proposes a binary class Bayesian feature selection framework labeling features as markers or non-markers [12, 13, 15, 16]. Given data the sample-conditioned probability of each feature being a marker is found. Assuming independent features, Optimal Bayesian Filter (OBF) is obtained, and closed form posteriors for binary class problems assuming Gaussian [13] and categorical [43] features have been derived. While OBF assesses each feature individually, several suboptimal algorithms that account for marker dependencies are proposed in $[12,15,16]$ for binary class problems. This framework has also been extended to consider missing data in binary class problems [40]. Recent work presents a simple multiclass extension of this framework [42] and obtains a multiclass extension of OBF; however, it does not take structures into account. It outputs a set of potential markers with distributional differences, but cannot determine where the distributional differences lie.

Here we extend the Bayesian framework of $[13,16]$ to account for marker structures, obtain the sample conditioned probability of a feature being a marker and satisfying a potential structure for the case of independent features, present optimal feature selection algorithms for the proposed model, which we call Structured OBF (SOBF), perform synthetic simulations to compare with other algorithms, and apply SOBF to cancer datasets. Many of the top

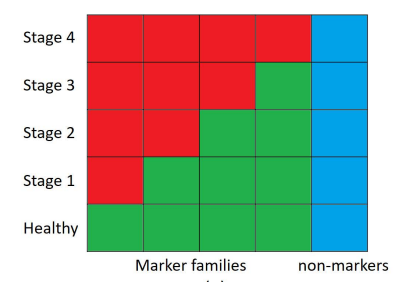

(a)

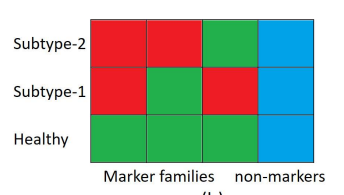

(b)
Figure 1: Possible structures for (a) cancer progression, and (b) two different subtypes of cancer.

genes and pathways are suggested to be involved in cancer. We also find potentially new biomarkers. The contribution is two fold: (1) individually weak markers that are affected in several classes are better detected, and (2) one can infer the structure of markers.

\section{FEATURE SELECTION MODEL}

Consider a multiclass feature selection problem with $C$ classes denoted by $\{0,1, \cdots, C-1\}$ and feature index set $F$. Assume features are markers or non-markers. Non-markers have the same distribution in all classes. A marker follows a distribution in a subset of classes, and another distribution in all other classes. For each feature $f$, pattern- 0 classes are those which follow a distribution similar to class 0 , and pattern-1 classes are those which do not follow the distribution of class 0 . We assume all pattern- 1 classes follow the same distribution, resulting in a binary pattern problem. We define a structure as a length $C$ vector denoting the pattern of each class. For example, a marker $f$ in column 2 of Fig. 1(a) has structure $S^{f}=[0,0,1,1,1]$, where the $c^{t h}$ element of $S^{f}$ is the pattern of class $c$. We denote the set of all possible marker structures by $S$, the true set of markers by $\bar{M}$, the true set of nonmarkers by $\bar{N}=F \backslash \bar{M}$, and the true structure of marker $f$ by $\bar{S} f$. Let $\pi(f)=P(f \in \bar{M})$ be the prior probability that $f$ is a marker, and assume the events $\{f \in \bar{M}\}$ are independent for all $f \in F$. Let $\pi\left(S^{f} \mid f\right)=P\left(\bar{S}^{f}=S^{f} \mid f \in \bar{M}\right)$ be the prior probability $S^{f}$ is the true structure of $f$ given $f \in \bar{M}$. Assume the events $\left\{\bar{S}_{i}=S^{f_{i}} \mid f_{i} \in \bar{M}\right\}$ and $\left\{\bar{S}_{j}=S^{f_{j}} \mid f_{j} \in \bar{M}\right\}$ are independent for all $f_{i}, f_{j} \in F$ such that $f_{i} \neq f_{j}$. Note $\pi\left(f, S^{f}\right)=P\left(f \in \bar{M}, \bar{S}^{f}=S^{f}\right)=\pi(f) \pi\left(S^{f} \mid f\right)$.

Now, fix $M \subseteq F$ as a potential set of markers. Let $N=F \backslash M$. Let $\pi(M)=P(\bar{M}=M)$ be the prior probability that $M$ is the true set of markers. Hence, $\pi(M)=\prod_{f \in M} \pi(f) \prod_{f \in N} 1-\pi(f)$. Let $\pi\left(S^{M} \mid M\right)=P\left(\bar{S}^{M}=S^{M} \mid \bar{M}=M\right)=\prod_{f \in M} \pi\left(S^{f} \mid f\right)$ be the prior probability that $\bar{S}^{M}$, the collection of true structures of all markers given $\bar{M}=M$, equals $S^{M}$, a collection of $S^{f}$ 's for all $f \in M$. $\pi\left(M, S^{M}\right)=\pi(M) \pi\left(S^{M} \mid M\right)$ is the prior probability that $M$ is the true set of markers and $S^{M}$ is the true structure of markers.

For a marker $f \in M$ let $\theta_{y}^{f}$ be the parameter describing the distribution of pattern- $y$ classes for $y=0,1$. For a non-marker $f \in N$ let $\theta^{f}$ describe its distribution in all classes. Let $\theta_{0}^{M}=$ $\left\{\theta_{0}^{f}: f \in M\right\}, \theta_{1}^{M}=\left\{\theta_{1}^{f}: f \in M\right\}$, and $\theta_{01}^{N}=\left\{\theta^{f}: f \in N\right\}$ be the collection of distribution parameters for pattern- 0 markers, pattern-1 markers, and non-markers, respectively. Let $\theta^{M}=$ $\left[\theta_{0}^{M}, \theta_{1}^{M}, \theta_{01}^{N}\right]$ be the collection of distribution parameters of pattern0 markers, pattern-1 markers, and non-markers assuming $M$ is the set of markers. Assume $\theta_{0}^{f_{1}}$,s, $\theta_{1}^{f_{1}}$,s, and $\theta^{f_{2}}$, s are independent for all $f_{1} \in M$ and $f_{2} \in N$ given $\bar{M}=M$. Hence, $\pi\left(\theta^{M} \mid M\right)=$ 
$\prod_{f \in M} \pi\left(\theta_{0}^{f}\right) \pi\left(\theta_{1}^{f}\right) \prod_{f \in N} \pi\left(\theta^{f}\right)$, where $\pi\left(\theta^{M} \mid M\right)$ is the prior on $\theta^{M}$ given $\bar{M}=M$, and $\pi\left(\theta_{y}^{f}\right)$ and $\pi\left(\theta^{f}\right)$ are the priors on $\theta_{y}^{f}$ and $\theta^{f}$ given $\bar{M}=M$, respectively. We assume $\theta^{M}$ and $S^{M}$ are independent given $\bar{M}=M$. Now, let $x$ be a sample point drawn from class $c$. Assuming features are independent, the marginal distribution of all features describes the joint distribution. Hence,

$$
f\left(x \mid M, S^{M}, \theta^{M}, c\right)=\prod_{f \in M} f\left(x^{f} \mid \theta_{y}^{f}\right) \prod_{f \in N} f\left(x^{f} \mid \theta^{f}\right),
$$

where $y=S^{f}[c]$ is the $c^{t h}$ element of structure vector $S^{f}, x^{f}$ denotes the value of sample point $x$ for feature $f$, and $f(. \mid \theta)$ is the density parametrized by $\theta$. Let $\mathcal{S}$ be a training sample of size $n$ where points in each pattern are independent and identically distributed (i.i.d.) and points in different patterns are independent of each other. The likelihood of observing sample $\mathcal{S}$ given $M, S^{M}$, and $\theta^{M}$ is $f\left(\mathcal{S} \mid M, S^{M}, \theta^{M}\right)=\prod_{c=0}^{C-1} \prod_{x \in \mathcal{S}_{c}} f\left(x \mid M, S^{M}, \theta^{M}, c\right)$, where $\mathcal{S}_{c}$ is the portion of training sample in class $c$. Therefore,

$$
f\left(\mathcal{S} \mid M, S^{M}\right)=\int \pi\left(\theta^{M} \mid M\right) f\left(\mathcal{S} \mid M, S^{M}, \theta^{M}\right) d \theta^{M},
$$

is the likelihood of $\mathcal{S}$ given $M$ and $S^{M}$. Recall $\theta^{M}$ and $S^{M}$ are independent given $M$. Following steps in $[15,16]$ we have the posterior

$$
\begin{aligned}
\pi^{*}\left(M, S^{M}\right) & =P(\bar{M}=M \mid \mathcal{S}) P\left(\bar{S}^{M}=S^{M} \mid \bar{M}=M, \mathcal{S}\right) \\
& \propto \pi\left(M, S^{M}\right) \prod_{f \in N} \int \pi\left(\theta^{f}\right) \prod_{x \in \mathcal{S}} f\left(x^{f} \mid \theta^{f}\right) d \theta^{f} \\
& \times \prod_{y=0}^{1} \prod_{f \in M} \int \pi\left(\theta_{y}^{f}\right) \prod_{x \in \mathcal{S}_{y}(f)} f\left(x^{f} \mid \theta_{y}^{f}\right) d \theta_{y}^{f},
\end{aligned}
$$

where $\mathcal{S}_{y}(f)$ is the portion of $\mathcal{S}$ comprised of points in classes that follow pattern $y$ for feature $f$. Further simplification gives $\pi^{*}\left(M, S^{M}\right) \propto \prod_{f \in M} h\left(f, S^{f}\right)$, where $h\left(f, S^{f}\right)$ is

$$
\frac{\pi(f) \pi\left(S^{f} \mid f\right)}{1-\pi(f)} \times \frac{\prod_{y=0}^{1} \int \pi\left(\theta_{y}^{f}\right) \prod_{x f \in \mathcal{S}_{y}^{f}} f\left(x^{f} \mid \theta_{y}^{f}\right) d \theta_{y}^{f}}{\int \pi\left(\theta^{f}\right) \prod_{x f \in \mathcal{S} f} f\left(x^{f} \mid \theta^{f}\right) d \theta^{f}} .
$$

The posterior probability that $f$ is a marker with structure $S^{f}$ is

$$
\begin{aligned}
\pi^{*}\left(f, S^{f}\right) & =P(f \in \bar{M} \mid \mathcal{S}) P\left(\bar{S}^{f}=S^{f} \mid f \in \bar{M}, \mathcal{S}\right) \\
& =\sum_{M \subseteq F \backslash\{f\}} \sum_{S^{M}} \pi^{*}\left(M \cup\{f\}, S^{M \cup\{f\}}\right) \\
& =\frac{\sum_{M \subseteq F \backslash\{f\}} \sum_{S^{M}} h\left(f, S^{f}\right) \prod_{f^{\prime} \in M} h\left(f^{\prime}, S^{f^{\prime}}\right)}{\sum_{M \subseteq F} \sum_{S^{M}} \prod_{f^{\prime} \in M} h\left(f^{\prime}, S^{f^{\prime}}\right)},
\end{aligned}
$$

where the sums over $S^{M}$ consider all possible structures of $M$, $S^{M}=\left\{S^{\prime}: f^{\prime} \in M\right\}$, sums over $S^{\prime}$ consider all $S^{\prime} \in S$, and $S^{M \cup\{f\}}$ is the structure of feature set $M \cup\{f\}$. We decompose the denominator into terms that contain $h\left(f, S^{\prime}\right)$ for some $S^{\prime} \in S$, and those that do not. After factoring the numerator and denominator, we obtain:

$$
\begin{aligned}
\pi^{*}\left(f, S^{f}\right) & =\frac{h\left(f, S^{f}\right) \sum_{M \subseteq F \backslash\{f\}} \sum_{S^{M}} \prod_{f^{\prime} \in M} h\left(f^{\prime}, S^{f^{\prime}}\right)}{\left(1+\sum_{S^{\prime}} h\left(f, S^{\prime}\right)\right) \sum_{M \subseteq F \backslash\{f\}} \sum_{S^{M}} \prod_{f^{\prime} \in M} h\left(f^{\prime}, S^{f^{\prime}}\right)} \\
& =\frac{h\left(f, S^{f}\right)}{1+\sum_{S^{\prime}} h\left(f, S^{\prime}\right)},
\end{aligned}
$$

Note that the posterior probability that $f$ is a marker is

$$
\pi^{*}(f)=P(f \in \bar{M} \mid \mathcal{S})=\sum_{S f \in S} \pi^{*}\left(f, S^{f}\right) .
$$

\subsection{Gaussian Model}

Here we solve the proposed model for Gaussian features. Let $f$ be a marker with structure $S^{f}$. Hence, $\theta_{y}^{f}=\left[\mu_{y}^{f}, \sigma_{y}^{f}\right]$, where $\mu_{y}^{f}$ and $\sigma_{y}^{f}$ are the mean and variance of the Gaussian distribution of $f$ for pattern- $y$ classes. We assume a normal-inverse-Wishart prior on distribution parameters, i.e., $\pi\left(\theta_{y}^{f}\right)=\pi\left(\sigma_{y}^{f}\right) \pi\left(\mu_{y}^{f} \mid \sigma_{y}^{f}\right)$, where

$$
\begin{aligned}
\pi\left(\sigma_{y}^{f}\right) & =K_{y}^{f}\left(\sigma_{y}^{f}\right)^{-0.5\left(\kappa_{y}^{f}+2\right)} \exp \left(-0.5 s_{y}^{f} / \sigma_{y}^{f}\right), \\
\pi\left(\mu_{y}^{f} \mid \sigma_{y}^{f}\right) & =L_{y}^{f}\left(\sigma_{y}^{f}\right)^{-0.5} \exp \left(-0.5 v_{y}^{f}\left(\mu_{y}^{f}-m_{y}^{f}\right)^{2} / \sigma_{y}^{f}\right) .
\end{aligned}
$$

$s_{y}^{f}, \kappa_{y}^{f}, m_{y}^{f}$, and $v_{y}^{f}$ are hyperparameters, which are assumed given and fixed. $s_{y}^{f}$ is the scale parameter and $\kappa_{y}^{f}$ is the degrees of freedom of the inverse-Wishart distribution. For a proper prior $s_{y}^{f}, \kappa_{y}^{f}>0$. If $\kappa_{y}^{f}>2$, then $E\left(\sigma_{y}^{f}\right)=s_{y}^{f} /\left(\kappa_{y}^{f}-2\right) . m_{y}^{f}$ describes the average mean of feature $f$ and for a proper prior we need $v_{y}^{f}>0 . K_{y}^{f}$ and $L_{y}^{f}$ represent the relative weights of each distribution. For a proper distribution we have $K_{y}^{f}=\left(s_{y}^{f}\right)^{0.5 \kappa_{y}^{f}} 2^{-0.5 \kappa_{y}^{f}} / \Gamma\left(0.5 \kappa_{y}^{f}\right)$ and $L_{y}^{f}=\left(2 \pi / v_{y}^{f}\right)^{-0.5}$, where $\Gamma$ denotes the gamma function. These hyperparameters incorporate prior information, expert knowledge, and experimental constraints; however, in absence of reliable prior information non-formative priors can be used, which have been shown to enjoy robust performance across large families of distribution parameters $[15,16,42]$. Note extra care needs to be taken to ensure $\pi^{*}(f)$ exists when non-informative priors are utilized.

Given training sample, the posterior distribution on $\theta_{y}^{f}$ is again normal-inverse-Wishart with updated hyperparameters: $\kappa_{y}^{f *}=\kappa_{y}^{f}+$ $n_{y}\left(S^{f}\right), v_{y}^{f *}=v_{y}^{f}+n_{y}\left(S^{f}\right), m_{y}^{f *}=\frac{v_{y}^{f} m_{y}^{f}+n_{y}\left(S^{f}\right) \hat{\mu}_{y}^{f}}{v_{y}^{f^{*}}}$, and

$$
s_{y}^{f *}=s_{y}^{f}+\left(n_{y}\left(S^{f}\right)-1\right) \hat{\sigma}_{y}^{f}+v_{y}^{f} n_{y}\left(S^{f}\right)\left(\hat{\mu}_{y}^{f}-m_{y}^{f}\right)^{2} / v_{y}^{f *},
$$

where $\hat{\mu}_{y}^{f}, \hat{\sigma}_{y}^{f}$ and $n_{y}\left(S^{f}\right)$ are sample mean, sample variance, and the number of points in all classes $c$ whose $S^{f}[c]=y$, respectively.

Now assume $f$ is a non-marker. Hence, the Gaussian distribution of $f$ is parametrized by $\theta^{f}=\left[\mu^{f}, \sigma^{f}\right]$, where $\mu^{f}$ and $\sigma^{f}$ are the mean and variance, respectively. We again assume the prior on $\theta^{f}$ is normal-inverse-Wishart with hyperparameters $s^{f}, \kappa^{f}, m^{f}$, and ${ }_{v}^{f}$, and relative weights $K^{f}$ and $L^{f}$. Given data the posterior on $\theta^{f}$ is again normal-inverse-Wishart with updated hyperparameters $\kappa^{f *}=\kappa^{f}+n, v^{f *}=v^{f}+n, m^{f *}=\frac{v^{f} m^{f}+n \hat{\mu}^{f}}{v^{f *}}$, and

$$
s^{f *}=s^{f}+(n-1) \hat{\sigma}^{f}+v^{f} n\left(\hat{\mu}^{f}-m^{f}\right)^{2} / v^{f *},
$$

where $\hat{\mu}^{f}$ and $\hat{\sigma}^{f}$ are sample mean and variance all training points, respectively. Further simplification gives

$$
h\left(f, S^{f}\right)=Q^{f}\left(\frac{\left(s_{0}^{f *}\right)^{\kappa_{0}^{f *}}\left(s_{1}^{f *}\right)^{\kappa_{1}^{f *}}}{\left(s^{f *}\right)^{\kappa^{f *}}}\right)^{-0.5},
$$


where

$$
\begin{aligned}
Q^{f} & =\frac{K_{0}^{f} L_{0}^{f} K_{1}^{f} L_{1}^{f}}{K^{f} L^{f}}\left(\frac{\left(s_{0}^{f}\right)^{\kappa_{0}^{f}}\left(s_{1}^{f}\right)^{\kappa_{1}^{f}}}{\left(s^{f}\right)^{\kappa^{f}}}\right)^{0.5}\left(\frac{v_{0}^{f *} v_{1}^{f *}}{2 \pi v^{f *}}\right)^{-0.5} \\
& \times 2^{0.5\left(\kappa_{0}^{f *}+\kappa_{1}^{f *}-\kappa^{f *}\right)} \times \frac{\Gamma\left(0.5 \kappa_{0}^{f *}\right) \Gamma\left(0.5 \kappa_{1}^{f *}\right)}{\Gamma\left(0.5 \kappa^{f *}\right)} .
\end{aligned}
$$

Note under non-informative priors we need $s_{y}^{f}, s^{f}, \kappa_{y}^{f}, \kappa^{f}, v_{y}^{f}, v^{f}>$ 0 to insure $\pi^{*}(f)$ exists.

\subsection{Set and Structure Selection}

Given $\pi^{*}(f)$ for all features $f$ one set needs to be declared as the set of markers. Maximum Number Correct (MNC) criterion maximizes the expected number of correctly labeled features $[12,14]$ and Constrained MNC (CMNC) maximizes the expected number of correctly labeled features subject to declaring $D$ features as markers, where $D$ is a parameter set by the user [15]. The solution of MNC is

$$
G^{M N C}=\left\{f: \pi^{*}(f)>0.5\right\},
$$

and the solution of CMNC is to rank features by $\pi^{*}(f)$ and pick the top $D$ features $[15,16]$. For the proposed model, for each feature $f$, $\mathcal{S}^{f}$, the portion of sample corresponding to feature $f$, is sufficient to obtain $\pi^{*}(f)$. Hence, we have obtained the structured multiclass extension of OBF, hereafter denoted by Structured OBF (SOBF).

Given $\pi^{*}\left(f, S^{f}\right)$ one can estimate the underlying structure of markers. However, as many practical applications demand, such inference should be made only when one is confident of the underlying structure. In other words, the cost of assigning a wrong structure to a marker is larger than assigning no structure at all. This motivated us to propose the Structure Assignment-SOBF (SA-SOBF) algorithm. ${ }^{1}$ For each feature $f$, (1) if $\pi^{*}\left(f, S^{f}\right) \geq T$ where threshold $T \in(0,1)$ and $S^{f}=\arg \max _{S^{\prime} \in S} \pi^{*}\left(f, S^{\prime}\right)$, then $f$ is declared a marker with structure $S^{f}$. (2) If $\pi^{*}(f) \geq T$ but $\pi^{*}\left(f, S^{\prime}\right)<T$ for all $S^{\prime} \in S$, then $f$ is declared a marker without a reported structure. Finally, (3) if $\pi^{*}(f)<T$ then $f$ is labeled as a non-marker. For $T=0.5$, MNC-SOBF and SA-SOBF output the same sets, except that SA-SOBF identifies the structure of strong markers as well. Increasing $T$ increases sensitivity at the expense of specificity, i.e., the selected features are more likely to be markers, while it's more likely that more markers will be missed.

\section{SIMULATIONS}

Here we provide synthetic simulations based on the proposed model and a model developed to mimic microarrays to compare SOBF with other popular feature selection algorithms.

\subsection{Bayesian Simulations}

Assume $|F|=5070,|\bar{M}|=70$, and $C=4$. With 4 classes there are $|S|=7$ possibilities for the structure of a marker. We assume there are 10 markers for each possible structure. We first randomly pick $|\bar{M}|$ features as markers and assign their structures. Then, we randomly draw distribution parameters of each feature from a normal-inverse-Wishart distribution with hyperparameters $s_{0}^{f}=s_{1}^{f}=s^{f}=0.5, \kappa_{0}^{f}=\kappa_{1}^{f}=\kappa^{f}=3, m_{0}^{f}=m_{1}^{f}=m^{f}=0$, and

${ }^{1}$ A MATLAB implementation of SOBF and SA-OBF is provided on github $v_{0}^{f}=v_{1}^{f}=v^{f}=3$. Given distribution parameters a stratified sample with an equal number points in each class is drawn. Given the training sample MNC-SOBF, CMNC-SOBF, MNC-OBF, CMNC-OBF, ANOVA picking top $|\bar{M}|$, ANOVA with False Discovery Rate (FDR) correction denoted by ANOVA(FDR), mutual information (MI) [45], FOHSIC [53], ReliefF [29], and Eigenvector Centrality Feature Selection (ECFS) [45] declare their set of markers. MNC-OBF and $\mathrm{CMNC}-\mathrm{OBF}$ are the unstructured versions of OBF proposed in [42]. The outputs of feature selection algorithms and true markers are saved. CMNC-SOBF, CMNC-OBF, ANOVA, MI, FOHSIC, ReliefF, and ECFS output $|\bar{M}|$ features, and ANOVA(FDR), MNC-SOBF, and MNC-OBF output a variable number of features. ANOVA(FDR) uses Benjamini-Hochberg procedure (BH) [3] to bound FDR by $5 \%$. We first run a first phase filtration by ANOVA picking the top 250 features which are then used with FOHSIC, ReliefF, and ECFS to pick $|\bar{M}|$ markers. We implemented a first phase filtration due to extensive computation cost of these algorithms. ReliefF uses 5 nearest neighbors. ECFS has tuning parameter $\alpha$. We test for all $\alpha \in\{0.1,0.2, \cdots, 5\}$, and for each sample size use the value that resulted in the largest average number of correctly labeled features.

SOBF and OBF assume $\pi(f)=|\bar{M}| /|F|$, and SOBF assumes $\pi\left(S^{f} \mid f\right)=1 /|S|$. We use the proper normal-inverse-Wishart prior (PP) used to generate data and Jeffreys non-informative prior (JP). JP assumes $K_{0}^{f} L_{0}^{f}=K_{1}^{f} L_{1}^{f}=K^{f} L^{f}=0.01$, and all other hyperparameters are zero. SOBF uses eqs. (4) and (5) to find $h\left(f, S^{f}\right)$ and uses eq. (2) to find $\pi^{*}\left(f, S^{f}\right)$ for all $f$ and $S^{f}$, then uses eq. (3) to find $\pi^{*}(f)$ for all $f$. OBF uses the model of [42] to obtain its own $\pi^{*}(f)$. MNC-SOBF and MNC-OBF use eq. 6, and CMNC-SOBF and CMNC-OBF output the top $|\bar{M}|$ features with largest $\pi^{*}(f)$.

Figures 2(a) and 2(b) plot the average number of correctly labeled features over 1000 iterations as sample size increases from 20 to 200 in steps of 20. Among methods that output a variable number of features MNC-SOBF-PP performs superior and is closely followed by MNC-OBF-PP and MNC-SOBF-JP. Among methods that output a fixed number of markers CMNC-SOBF-PP performs superior and is closely followed by CMNC-SOBF-JP and CMNC-OBF-PP. Note OBF experiences a sharper drop in performance compared with SOBF when JP is used instead of PP. We observed SOBF enjoys better performance than $\mathrm{OBF}$ as it better detects weak markers, i.e., markers whose $\mu_{0}^{f}$ and $\mu_{1}^{f}$, and $\sigma_{0}^{f}$ and $\sigma_{1}^{f}$ are close to each other; however, note that SOBF is optimal under the current data generation model. While ANOVA(FDR) performs very well under small sample sizes, it typically outputs very few features compared with other algorithms. Note outputting the empty set correctly labels all 5000 non-markers. Table 1 compares runtime of the different algorithms used for feature selection when $n=200$ and the runtime of SOBF in taken as the unit of time. The choice of prior (PP or JP) and the set selection criterion (MNC or CMNC) does not affect the runtime of $\mathrm{OBF}$ and SOBF very much. As Tab. 1 suggests, SOBF is much faster than many popular methods of analysis such as ANOVA. While OBF is much faster than SOBF, as long as the number of possible structures to evaluate is not very large, the additional runtime of SOBF is typically acceptable in practical settings.

Now we evaluate how SA-SOBF performs with $T=0.5$. Among the methods of previous simulation only ANOVA can be used to infer the underlying structure of a marker. We use MATLAB's 
Table 1: Runtime comparison of the Bayesian simulation selection algorithms relative to SOBF when $n=200$

\begin{tabular}{|c|c|c|c|c|c|c|c|}
\hline Algorithm & SOBF & OBF & ANOVA & MI & ReliefF & FOHSIC & ECFS(best) \\
\hline Runtime & 1 & 0.1 & 170 & 100 & 30 & 800 & 2500 \\
\hline
\end{tabular}

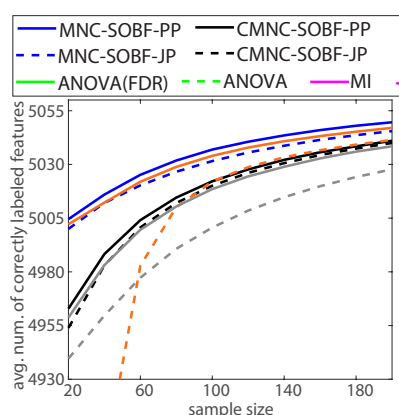

(a)

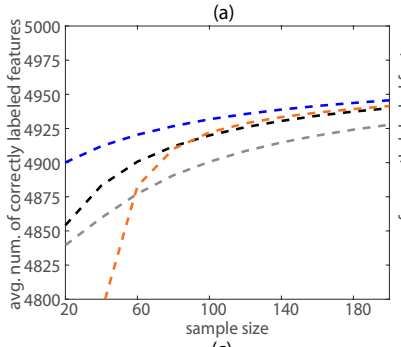

(c)

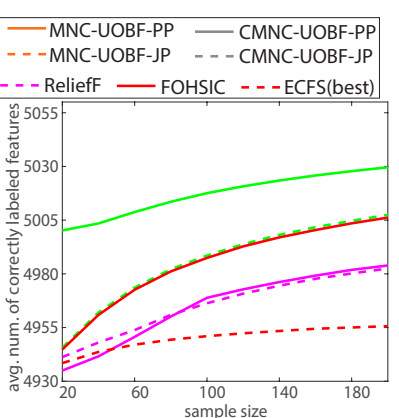

(b)

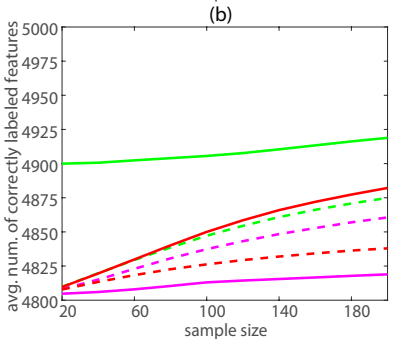

(d)
Figure 2: Average number of correctively labeled features versus sample size for random distribution parameters using (a) SOBF and OBF, and (b) ANOVA, MI, ReleifF, FOHSIC, and ECFS. Average number of correctively labeled features versus sample size for microarray model using (c) SOBF and OBF, and (d) ANOVA, MI, ReleifF, FOHSIC, and ECFS.

built-in multiple comparison function multcompare inputted with ANOVA's feature set using Tukey's honest significant difference criterion (HSD), Bonferroni method, Dunn and Sidák's approach (D\&S), Scheffé's S procedure (Sproc.), and Fisher's least significant difference procedure (LSD). We sum the number of correctly labeled non-markers, and the number of markers whose structures are correctly found as the performance metric. Figure 3(a) plots performance as sample size increases from 20 to 200 in steps of 200 over 1000 iterations. SA-SOBF-JP and SA-SOBF-PP outperform ANOVA with multiple comparisons. Note the performance metric of Fig. 3(a) does not consider the number of features correctly labeled as markers without any assigned structures. This is why JP outperforms PP in Fig. 3(a).

\subsection{Synthetic Microarray Model}

Here we generalize a synthetic model developed to mimic microarrays to better evaluate SOBF under realistic settings where its assumptions might be violated. The original binary class model was proposed in [23] and has been extended in [15]. We generalize the model of [15] for structured multiclass data. In short, we use class $y$ distribution parameters of the binary class model for pattern- $y$ markers. In this model features are either markers or non-markers. Markers are either global or heterogeneous. Global markers are homogeneous within each pattern, construct blocks of size $k$, all markers within each block have the same structure, and

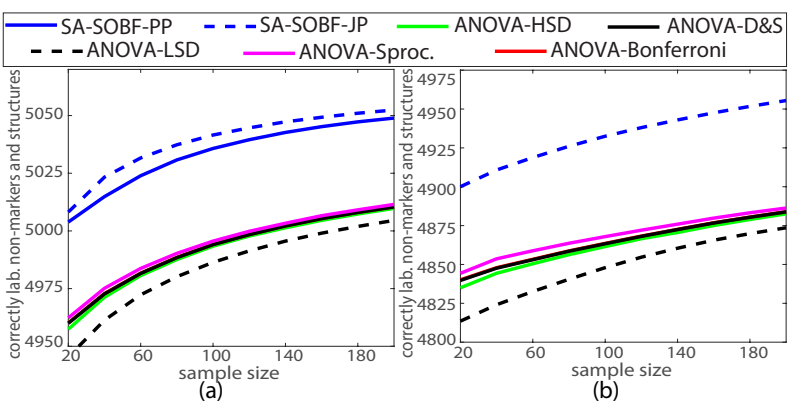

Figure 3: Average number of correctively detected nonmarkers and marker structures versus sample size for (a) randomly generated distribution parameters, and (b) synthetic microarray model data.

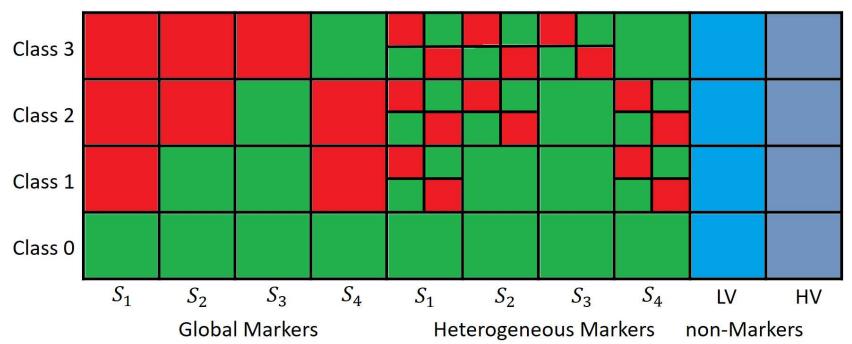

Figure 4: An example of generalized synthetic model for a 4 class problem and structures $S_{1}, S_{2}, S_{3}$, and $S_{4}$.

pattern- $y$ global markers are jointly Gaussian. The mean vector of a pattern- 0 block is $\mu_{0}=[0, \cdots, 0]$, and pattern- 1 markers are of three types: redundant, synergetic, and marginal, with mean vectors $\mu_{1}=[1, \cdots, 1], \mu_{1}=[1,1 / 2, \cdots, 1 / k]$, and $\mu_{1}=[1,0, \cdots, 0]$, respectively in each block. Each block of pattern- $y$ global markers has covariance matrix $\Sigma_{y}=\sigma_{y} \Sigma_{y}$, where diagonal elements of $\Sigma_{y}$ are 1 and off-diagonal elements are $\rho_{y} . \sigma_{y}$ and $\rho_{y}$ are parameters of the model. Heterogeneous markers also construct size $k$ blocks. Pattern-0 heterogeneous markers are similar to pattern-0 global markers, and in each pattern-1 class construct $\tilde{c}$ subclasses. One of the subclasses follows a distribution similar to pattern-1 global markers, and the remaining subclasses follow a distribution similar to pattern-0 markers. Non-markers are either low variance (LV) or high variance (HV). LV non-markers are similar to pattern-0 markers. HV non-markers are independent, and follow a Gaussian mixture with distribution $p N\left(0, \sigma_{0}\right)+(1-p) N\left(1, \sigma_{1}\right)$, where $p$ is drawn from the uniform distribution over $[0,1]$. Figure 4 provides an example for a 4 class problem with structures $S_{1}=[0,1,1,1]$, $S_{2}=[0,0,1,1], S_{3}=[0,0,0,1]$, and $S_{4}=[0,1,1,0]$, and $\tilde{c}=2$, where each row is a class and each column is a family of features.

For our multiclass simulation we assume $C=4$. Fix $|F|=5000$ and $|\bar{M}|=100$, with 20 global and 80 heterogeneous synergetic markers, and $2000 \mathrm{HV}$ non-markers. We assume $\tilde{c}=2$ and 4 structures similar to Fig. 4. We also assume $k=5, \sigma_{0}=0.25, \sigma_{1}=0.64$, and $\rho_{0}=\rho_{1}=0.9$. Among the 4 blocks of global markers one block follows each of the structures, and among 16 blocks of heterogeneous markers 4 blocks follow each of the structures. Given distribution parameters, a stratified sample with equal number of points in each class is drawn. We implement all feature selection 
algorithms of sec. 3.1 except SOBF-PP and OBF-PP under MNC and CMNC. We assume $\pi(f)=|\bar{M}| /|F|$ and $\pi\left(S^{f} \mid f\right)=0.25$. Figures 2(c) and 2(d) plot the average number of correctly labeled features as sample size increases from 20 to 200 in steps of 20 over 1000 iterations. MNC-SOBF-JP outperforms other algorithms and is followed by MNC-OBF-JP. CMNC-SOBF-JP outperforms algorithms that output $|\bar{M}|$ features. Under small samples, MNC-OBF-JP performs inferior to CMNC-OBF-JP as it labels too many features as markers. Note the constraint of CMNC to output $|\bar{M}|$ features limits the number of false discoveries, i.e., labeling non-markers as markers. However, for larger sample sizes MNC-OBF-JP outperforms CMNC-OBF-JP. While MNC-OBF-JP enjoys competitive performance compared with MNC-SOBF-JP for large samples, it only labels features as markers or non-markers, and cannot detect their underlying structure.

We now perform a simulation similar to the second simulation of sec. 3.1, except we only implement SA-SOBF under JP. Figure 3(b) plots the average number of correctly detected non-markers and marker structures. Again SA-SOBF-JP outperforms ANOVA. While a marker might follow more than 2 patterns in practice, here we only consider binary pattern markers. We leave studying robustness of SOBF to structure mismatches for future work.

\section{REAL DATA}

We apply SOBF to two cancer datasets. Our goal is not only to find potentially new biomarkers or make hypotheses about their structure, but to verify that SOBF indeed finds biomarkers in real-world settings. Hence, we chose cancers that are extensively studied in the literature to better evaluate outputs of SOBF. We study top genes of SOBF and perform enrichment analysis using PANTHER (version 11) $[34,35]$. Many of the top SOBF genes and pathways are shown or suggested to be involved in the cancer under study, suggesting SOBF can indeed detect biomarkers. SOBF detects potentially new biomarkers as well. We use JP with hyperparameters similar to sec. 3.1, fix $\pi(f)=0.01$, and set $\pi\left(S^{f} \mid f\right)=1 /|S|$ for each dataset. We perform the following pipeline: We (1) remove probes which do not map to any genes, (b) perform feature selection to find a high-profile set of probes, and (c) among probes which map to the same gene sets, only the probe ranking highest is retained.

\subsection{Colon Cancer}

Data obtained in [52] is deposited in Gene Expression Omnibus (GEO) [8] with accession number GSE17538 and contains 238 points. $28,72,76$ and 56 points belong to stage $1,2,3$, and 4 patients, respectively. 6 points that belong to adenoma patients are not included in our analysis. As data contains patients in different stages of colon cancer, the goal is to find biomarkers that are involved in cancer progression. ANOVA using BH procedure to bound FDR by 0.1 declares only 7 significant probes. Labeling stage 1 and 2 patients as early stage cancer patients, and stage 3 and 4 patients as late stage, Welsch t-statistic reports 63 significant probes after bounding FDR by 0.1 using $\mathrm{BH}$ procedure. This implies there are potential biomarkers that ANOVA missed. We implement SOBF with structures $S_{1}=[0,1,1,1], S_{2}=[0,0,1,1]$, and $S_{3}=[0,0,0,1]$. A marker with structure $S_{i}$ follows one distribution until stage $i$, and another distribution at later stages. Comparing $\pi^{*}(f)$ with 0.9
Table 2: Top 21 colon cancer genes with structure $S_{1}$

\begin{tabular}{|l|l|l|l|l|l|}
\hline Rank & Gene & Rank & Gene & Rank & Gene \\
\hline 1 & C1orf173 & 8 & CTNNA2 & 15 & ATF6B \\
2 & GAGE1,4-7,12 & 9 & STAR & 16 & PCDHB6 \\
3 & DCC & 10 & CEACAM5 & 17 & CT45A1-6 \\
4 & GAGE1,2,4-8,12 & 11 & GPM6A & 18 & LOC100507186 \\
5 & KRT31 & 12 & ASB5 & 19 & SNAP25 \\
6 & LOC100133920,286297 & 13 & NOL4 & 20 & CPS1 \\
7 & S100A7 & 14 & DAZ1-4 & 21 & SNAP91 \\
\hline
\end{tabular}

SOBF-JP selects 499 probes mapping to 437 different genes. While MNC compares $\pi^{*}(f)$ with 0.5 , we used a larger threshold to report genes for which the data suggests are biomarkers with probability of at least $90 \%$. We have increased $T$ to increase sensitivity and have more confidence on the identity of reported markers; however, a larger number weak markers might be missed compared to $T=0.5$. SA-SOBF-JP with $T=0.9$ outputs the same feature set as SOBF-JP, and the structure of 479 probes mapping to 420 different genes, i.e., genes which we can label as a biomarker and be confident in their structure with probability of at least $90 \%$.

We now study top genes of each structure in more detail. Table 2 lists the top SOBF-JP genes with structure $S_{1}$. The top gene, c1orf173, has been identified as a biomarker in [9] studying metastatic colon cancer to the lung. c1orf173 was "validated by targeted deep sequencing using the Ion Torrent PGM customized AmpliSeq panel” [9]. c1orf173 is identified as a potential biomarker in [33] as well. Cancer/Testis (CT) antigens, such as GAGE genes, have been suggested to be involved in several types of cancer, promote cancer progression, and are candidates for immunotherapy [18, 50]. CT antigens are more prevalent in melanoma and ovarian cancers compared with colon cancer [4]. Here GAGE genes are over-expressed among a subpopulation of late stage cancer patients, suggesting GAGE genes might be involved in colon cancer progression. CT45 genes are also CT genes that are suggested to be involved in cancer [18]. Recent studies show that "a number of CTAs promote epithelialmesenchymal transition (EMT) and genesis of cancer stem like cells, escalating tumorigenesis, invasion, and metastasis" [59]. Here we observe CT45 genes are over-expressed in late stage patients. Indeed, if a gene is involved in cancer progression and metastasis, we expect it to be affected to a higher extent in late stage patients. We observe DCC, which is shown to be deleted in many colon cancer patients [47], has lower expression in late stage patients. Histograms of GAGE genes is provided in Fig. 5, where the left and right columns are pattern- 0 and pattern- 1 classes, respectively.

Table 3 lists the top SOBF-JP genes with structure $S_{2}$. KRT13 [58], TTN [10], and SERPINB13 [20] are suggested to be involved in colon cancer. NKX2-1, also known as TTF-1, is suggested to be involved in many types of cancer such as lung cancer [26, 28]; however, its role in colon cancer requires further investigation. Histogram of NKX2-1 is provided in Fig. 5, where the left and right columns are pattern- 0 and pattern- 1 classes, respectively. CTAG1A and CTAG1B are CT genes that are suggested to be affected in colon cancer [27]. Table 4 lists top genes with structure $S_{3}$. While RFX6 is suggested to be involved in prostate cancer [24], its role in colon cancer requires further investigation. SERPINC1 is shown to be involved in colon cancer [38]. SLC2A2, also known as GLUT2 [31] is affected in colon cancer.

We use PANTHER to perform enrichment analysis using the 437 genes of SOBF-JP. PANTHER pathways recognize 67 genes, 
Table 3: Top 21 colon cancer genes with structure $S_{2}$

\begin{tabular}{|l|l|l|l|l|l|}
\hline Rank & Gene & Rank & Gene & Rank & Gene \\
\hline 1 & KRT13 & 8 & ATP5E & 15 & KIAA0087 \\
2 & TTN & 9 & FAM19A2 & 16 & SPRR2B \\
3 & NKX2-1 & 10 & STOML3 & 17 & TMPRSS11B \\
4 & SERPINB13 & 11 & PDK4 & 18 & CTAG2 \\
5 & CTAG1A,B & 12 & SCGB1A1 & 19 & KBTBD10 \\
6 & TRDN & 13 & KRT4 & 20 & FUT9 \\
7 & SFTA3 & 14 & ZIC1 & 21 & ACTA1 \\
\hline
\end{tabular}

Table 4: Top 21 colon cancer genes with structure $S_{3}$

\begin{tabular}{|l|l|l|l|l|l|}
\hline Rank & Gene & Rank & Gene & Rank & Gene \\
\hline 1 & RFX6 & 8 & APOA1 & 15 & DIO1 \\
2 & CPO & 9 & AHSG & 16 & SLC2A2 \\
3 & ABCA13 & 10 & GC & 17 & PDE6H \\
4 & SERPINC1 & 11 & ITIH2 & 18 & APOA4 \\
5 & PAQR9 & 12 & APOH & 19 & CFHR5 \\
6 & C17orf104 & 13 & FGA & 20 & HP HPR \\
7 & LOC339260 & 14 & APOC3 & 21 & SERPINA5 \\
\hline
\end{tabular}

Table 5: Top 10 enriched colon cancer pathways

\begin{tabular}{|l|l|l|l|}
\hline Rank & Pathway & P-value & Q-value \\
\hline 1 & Cadherin sig. p.w. & $7.88 \mathrm{E}-13$ & $1.28 \mathrm{E}-10$ \\
2 & Blood coagulation & $2.38 \mathrm{E}-08$ & $1.94 \mathrm{E}-06$ \\
3 & Plasminogen activating cascade & $3.96 \mathrm{E}-08$ & $2.15 \mathrm{E}-06$ \\
4 & Wnt sig. p.w. & $2.08 \mathrm{E}-07$ & $8.49 \mathrm{E}-06$ \\
5 & Nicotinic acetylcholine receptor sig. p.w. & $7.76 \mathrm{E}-03$ & $2.11 \mathrm{E}-01$ \\
6 & Cytoskeletal regulation by Rho GTPase & $1.17 \mathrm{E}-02$ & $2.72 \mathrm{E}-01$ \\
7 & ATP synthesis & $1.43 \mathrm{E}-02$ & $2.92 \mathrm{E}-01$ \\
8 & Beta1 adrenergic receptor sig. p.w. & $2.06 \mathrm{E}-02$ & $3.36 \mathrm{E}-01$ \\
9 & Beta2 adrenergic receptor sig. p.w. & $2.06 \mathrm{E}-02$ & $3.73 \mathrm{E}-01$ \\
10 & Triacylglycerol metabolism & $6.16 \mathrm{E}-02$ & $7.73 \mathrm{E}-01$ \\
\hline
\end{tabular}

which may result in reporting fewer significant enriched pathways. Bounding FDR by 5\% the top 4 pathways are significant. Table 5 lists the top 10 pathways. Many of the top pathways are shown to be involved in colon cancer, for instance, cadherin signaling [2, 39], blood coagulation [56], plasminogen activating cascade [17], and WNT signaling [44] pathways are shown to be affected in colon cancer. Furthermore, $\beta$ adrenergic signaling pathway, nicotine intake, and nicotinic acetylcholine receptors have been suggested to be affected in colon cancer $[5,57,60]$.

\subsection{Leukemia}

Data obtained in [22] is deposited on GEO with accession number GSE13204, containing 2096 points. 74, 542, 750, 76, and 206 points map to healthy people, and patients with different subtypes of Acute Myeloid Leukemia (AML), different subtypes of Acute Lymphoblastic Leukemia (ALL), Chronic Lymphocytic Leukemia (CLL), Chronic Myeloid Leukemia (CML), and Myelodysplastic syndrome (MDS), respectively. While the the data is pre-processed including a summarization and quantile normalization, we log normalize data so that it is more suitable for a Gaussian model.

We use SOBF-JP and consider all possible structures. Probes whose $\pi^{*}(f)>0.9$ map to 17769 genes. SA-SOBF-JP with $T=0.9$ outputs the structure of 15714 markers. Table 6 lists the top 60 genes with largest $\pi^{*}(f)$. Many of the top genes are shown or suggested to be involved in leukemia. For instance, DDR1, also known as CD167, is suggested to be involved in ALL [7]. SCARB1 is involved in AML [36], and [1] suggests NCRNA00258, also known as c21orf21, is only affected in CLL. With 6 classes there are 31 possible structures. Table 7 lists top 50 genes accounting for the subtypes they affect.

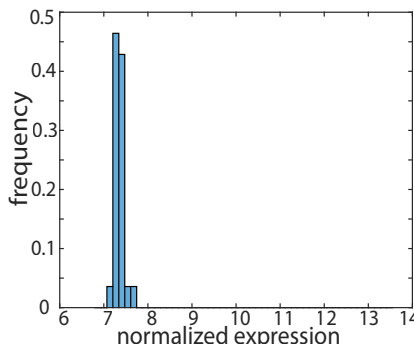

(a)

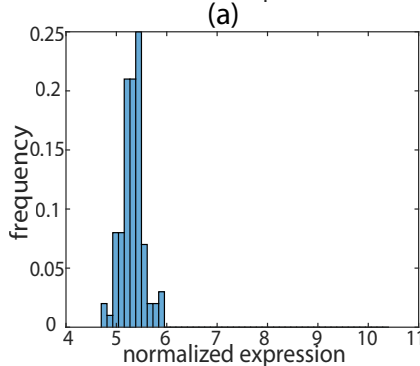

(c)

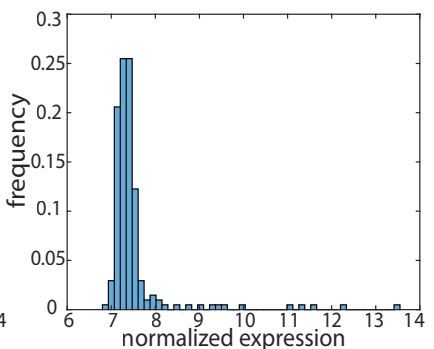

(b)

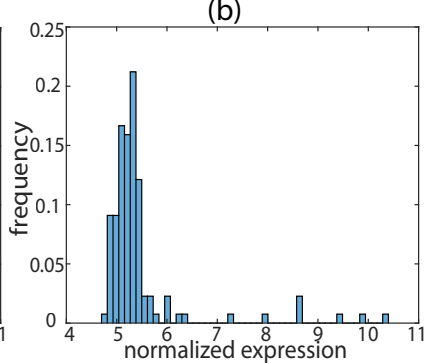

(d)
Figure 5: Colon cancer marker histogram of early stage patients for (a) GAGE genes and (c) NKX2-1, and histogram of late stage patients for (b) GAGE genes and (d) NKX2-1.

Not only do we detect high-profile genes as biomarkers, but we also make meaningful hypotheses on the leukemia subtypes they affect. Indeed a gene not listed as being affected in some subtype does not guarantee it not being affected in the subtype, but rather emphasizes that given the current observed data we cannot confidently declare if this gene is affected, or that it is affected to lesser extent compared with other subtypes. Figure 6 plots the histogram of top 2 genes illustrating they have extreme distributional differences for the estimated underlying structure. Top SOBF-JP genes affected only in certain subtypes of leukemia are high-profile biomarkers with extreme importance that require further investigation. Upon verification such markers can be used for various applications, such as predicting the risk of MDS progressing to AML, and developing drugs that target a specific or a broad range of leukemia subtypes. For instance, SCRAB1 and FCRL3 might be potential markers that can be used to differentiate between AML and MDS. A careful comparison of Fig. 6(c) suggests there might be another leukemia subtype for which SCARB1 is down-regulated and mixed with subtypes not affected in leukemia. Excluding AML and ALL, SASOBF-JP declares healthy people, MDS patients, and CML patients follow one distribution, and CLL patients follow another distribution. The histogram of SCARB1 for all classes is provided in Fig. 7. Comparing the histogram of CLL patients with other subtypes we conclude this inference is indeed correct. Further analysis provides the tree in Fig. 8 for a hierarchical clustering of leukemia subtypes based on expression of SCARB1. To obtain this hierarchy we started with all classes, used SA-SOBF-JP with $T=0.9$ to identify pattern-0 and pattern-1 classes, and again applied it to classes of each pattern separately. At each node if SA-SOBF-JP outputs a structure, we separate pattern- 0 and pattern- 1 classes as the children of current node; otherwise, we stop. These results indicate one of the shortcomings of the proposed binary pattern model when a marker follows several patterns. While SCARB1 is under-expressed in CLL patients, we missed it at first since we only consider 2 patterns. We 
Table 6: Top 60 genes of leukemia overall

\begin{tabular}{|l|l|l|l|l|l|l|l|}
\hline Rank & Gene & Rank & Gene & Rank & Gene & Rank & Gene \\
\hline 1 & DDR1 & 16 & TK1 & 31 & LOC100129196 & 46 & CANX \\
2 & SCARB1 & 17 & SUV39H2 & 32 & TTN & 47 & GLUL \\
3 & BEST4 & 18 & METTL8 & 33 & KIAA0895L & 48 & LDHA \\
4 & NCRNA00258 & 19 & TYMS & 34 & CLLU1 & 49 & P4HB \\
5 & ZNF101 & 20 & FILIP1L & 35 & ZBTB38 & 50 & CTSA \\
6 & TNFRSF13C & 21 & SNX25 & 36 & ZNF831 & 51 & CD63 \\
7 & FCRL3 & 22 & MCOLN2 & 37 & INO80C & 52 & PPP1CC \\
8 & CSF3R & 23 & AP1S3 & 38 & RUVBL2 & 53 & CTSD \\
9 & FAM129C & 24 & CDKN3 & 39 & FFAR1 & 54 & STMN1 \\
10 & CCDC141 & 25 & CD79A & 40 & CLNK & 55 & ZYX \\
11 & MGC16075 & 26 & SPIN3 & 41 & FCRL2 & 56 & LAMP2 \\
12 & NKX2-3 & 27 & ARPP21 & 42 & MGST1 & 57 & H2AFZ \\
13 & P2RY10 & 28 & LOC100289090 & 43 & DNTT & 58 & HDGF \\
14 & DPCD & 29 & GABRB2 & 44 & LOC440864 & 59 & CKAP4 \\
15 & MSRB3 & 30 & LOC285758 & 45 & ABCB4 & 60 & ANXA1 \\
\hline
\end{tabular}

Table 7: Top 50 genes of leukemia accounting for structures

\begin{tabular}{|l|l|l|l|l|l|}
\hline Rank & Gene & Subtype & Rank & Gene & Subtype \\
\hline 1 & DDR1 & ALL CLL & 26 & SPIN3 & CLL \\
2 & SCARB1 & AML ALL & 27 & ARPP21 & ALL \\
3 & BEST4 & CLL & 28 & LOC100289090 & CLL \\
4 & NCRNA00258 & CLL & 29 & GABRB2 & CLL \\
5 & ZNF101 & CLL & 30 & LOC285758 & ALL CLL \\
6 & TNFRSF13C & CLL & 31 & LOC100129196 & CLL \\
7 & FCRL3 & AML ALL & 32 & TTN & CLL \\
8 & CSF3R & ALL CLL & 33 & KIAA0895L & CLL \\
9 & FAM129C & ALL CLL & 34 & CLLU1 & AML ALL \\
10 & CCDC141 & CLL & 35 & ZBTB38 & CLL \\
11 & MGC16075 & CLL & 36 & ZNF831 & CLL \\
12 & NKX2-3 & AML MDS & 37 & INO80C & ALL \\
13 & P2RY10 & CLL & 38 & RUVBL2 & CLL \\
14 & DPCD & CLL & 39 & FFAR1 & ALL CLL \\
15 & MSRB3 & ALL CLL & 40 & CLNK & AML ALL \\
16 & TK1 & CLL & 41 & FCRL2 & CLL \\
17 & SUV39H2 & CLL & 42 & MGST1 & ALL CLL \\
18 & METTL8 & CLL & 43 & DNTT & ALL \\
19 & TYMS & AML ALL & 44 & LOC440864 & ALL \\
20 & FILIP1L & CLL & 45 & ABCB4 & CLL \\
21 & SNX25 & CLL & 46 & CANX & CLL \\
22 & MCOLN2 & CLL & 47 & GLUL & ALL CLL \\
23 & AP1S3 & CLL & 48 & LDHA & CLL \\
24 & CDKN3 & CLL & 49 & P4HB & ALL CLL \\
25 & CD79A & CLL & 50 & CTSA & ALL CLL \\
\hline
\end{tabular}

also missed that SCARB1 is over-expressed to a significantly larger extent in ALL compared with AML.

Finally, we perform enrichment analysis. We first use all genes detected as biomarkers, and then genes that are affected in AML to focus on pathways affected in AML. Note the latter analysis can also be done on any other subtype. Top 10 PANTHER pathways using all markers is provided in Tab. 8. Among all 17769 selected genes only 2290 (only $12.9 \%$ of selected genes) are recognized by PANTHER pathways. The small portion of genes recognized by PANTHER pathways affects enrichment analysis, increases p-values of enriched pathways, and greatly contributes to none of the results being significant. Furthermore, FDR correction algorithms are typically conservative, meaning some pathways in Tab. 8 could still be significant under $F D R<0.05$. Note the top genes not recognized by PANTHER pathways can be important cancer biomarkers whose interactions with other genes and the biological mechanisms they affect may provide invaluable insight on cancer biology. Note we still observe that many of the top pathways are suggested to be involved or affected in leukemia. For instance, PDGF signaling [11, 19], apoptosis [30, 37, 54], and CCKR signaling [6, 21] are suggested to be affected in several subtypes of leukemia. Changes in gonadotropin releasing hormone seems to be a side effect of radiation therapy in leukemia patients, in particular childhood leukemias $[46,51]$, suggesting the need for further verification and evaluation of potential biomarkers selected here for their clinical use. Table
Table 8: Top 10 enriched leukemia pathways

\begin{tabular}{|l|l|l|}
\hline Rank & Pathway & P-value \\
\hline 1 & Gonadotropin-releasing hormone receptor p.w. & $1.34 \mathrm{E}-02$ \\
2 & CCKR signaling map & $3.52 \mathrm{E}-02$ \\
3 & Apoptosis sig. p.w. & $4.06 \mathrm{E}-02$ \\
4 & EGF receptor sig. p.w. & $4.24 \mathrm{E}-02$ \\
5 & Integrin signalling p.w. & $4.84 \mathrm{E}-02$ \\
6 & PDGF sig. p.w. & $5.61 \mathrm{E}-02$ \\
7 & Inflam. mediated by chemokine \& cytokine sig. & $6.27 \mathrm{E}-02$ \\
8 & Wnt sig. p.w. & $8.99 \mathrm{E}-02$ \\
9 & Huntington disease & $9.91 \mathrm{E}-02$ \\
10 & B cell activation & $1.00 \mathrm{E}-01$ \\
\hline
\end{tabular}

Table 9: Top 10 enriched AML pathways

\begin{tabular}{|l|l|l|}
\hline Rank & Pathway & P-value \\
\hline 1 & Gonadotropin-releasing hormone receptor p.w. & $1.53 \mathrm{E}-03$ \\
2 & Cadherin sig. p.w. & $3.16 \mathrm{E}-03$ \\
3 & PDGF sig. p.w. & $3.31 \mathrm{E}-03$ \\
4 & Wnt sig. p.w. & $7.68 \mathrm{E}-03$ \\
5 & VEGF sig. p.w. & $1.53 \mathrm{E}-02$ \\
6 & Insulin/IGF p.w.-mitogen act. prot. kin. kin./MAP kin. & $2.39 \mathrm{E}-02$ \\
7 & CCKR signaling map & $3.09 \mathrm{E}-02$ \\
8 & FGF sig. p.w. & $4.06 \mathrm{E}-02$ \\
9 & EGF receptor sig. p.w. & $4.37 \mathrm{E}-02$ \\
10 & T cell activation & $4.43 \mathrm{E}-02$ \\
\hline
\end{tabular}

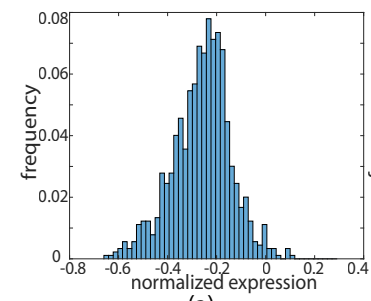

(a)

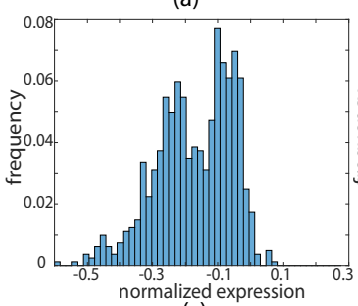

(c)

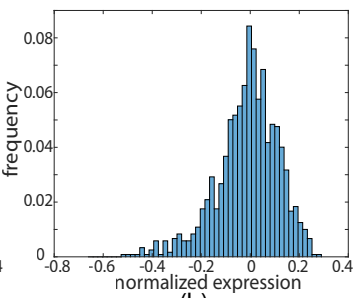

(b)

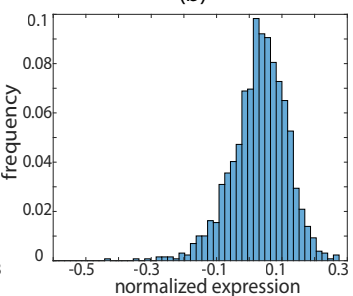

(d)
Figure 6: Leukemia marker histogram of healthy people and subtypes grouped with healthy people for (a) DDR1 and (c) SCARB1. Leukemia marker histogram of affected subtypes for (b) DDR1 and (d) SCARB1.

9 lists the top 10 enriched AML pathways, where among the 5057 genes detected as affected in AML only 635 (12.6\%) are recognized by PANTHER pathways. This again contributes to none of the enriched pathways being significant after FDR correction; however we still observe many of the top pathways are suggested to be affected in AML. We also observe that many top pathways are in common between Tabs. 8 and 9 .

\section{CONCLUSION AND FUTURE WORK}

Bayesian feature selection is a promising framework for biomarker discovery. This framework has been extended for block diagonal covariance models [16], Single Nucleotide Polymorphism (SNP) data [43], integrating prior information with data [41], and problems 

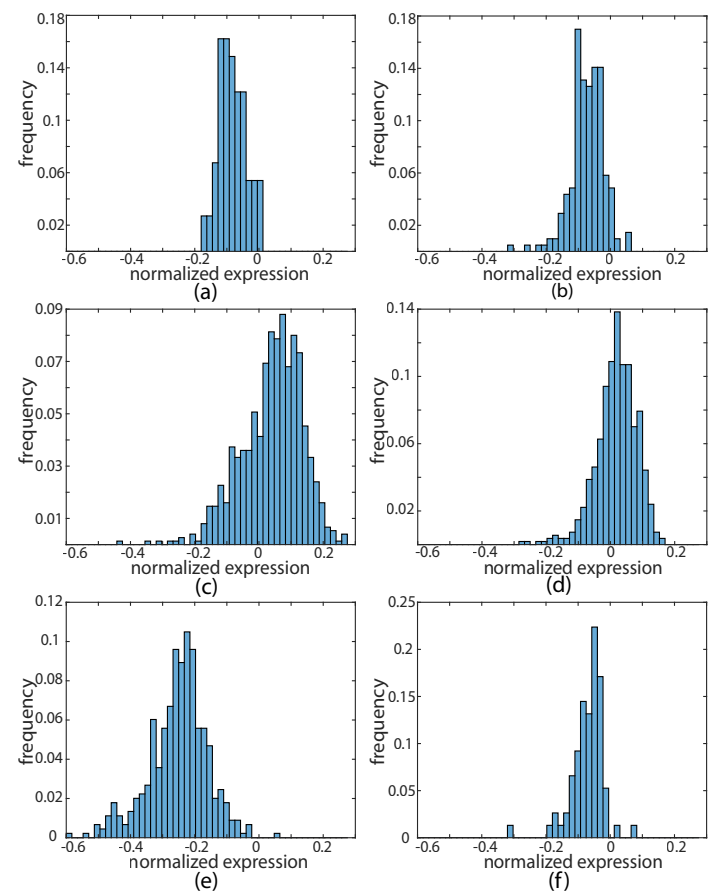

Figure 7: Histogram of SCARB1 for (a) healthy people, (b) MDS, (c) ALL, (d) AML, (e) CLL, and (f) CML patients.

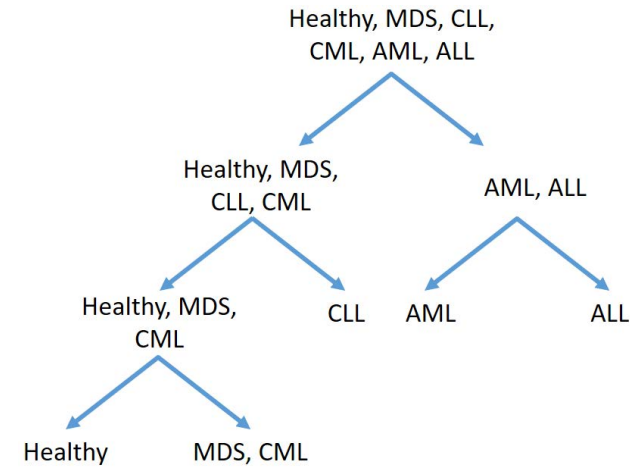

Figure 8: Hierarchical clustering of leukemia subtypes based on SCARB1 expression.

with missing data [40], suggesting its flexibility to adapt to the demands of the problem. Here we extended this framework to model structured multiclass problems, and illustrated how biomarker structures can be used to infer invaluable information about a potential biomarker. Applying SOBF to 2 cancer datasets we observed SOBF indeed detects biomarkers in a realistic setting, found potentially new biomarkers whose role in cancer require further investigation, and observed many interesting patterns in data, motivating new hypotheses about the role of certain biomarkers in cancer.

One extension for future work is to extend this model to account for marker dependencies. When feature sets are considered the number of possible structures to evaluate grows combinatorially and suitable suboptimal methods need to be developed. Extensions with more than 2 patterns should be considered. While the extension seems straightforward, (a) constructing a well-defined problem that all structures are identifiable is difficult, and (b) the number of structures to evaluate grows combinatorially, demanding suitable suboptimal algorithms. Sequences of binary pattern problems seem to be a good candidate to develop suboptimal methods for multipattern problems, as we observed for SCARB1 in sec. 4.2. Future work should study structure mismatch, i.e., cases where the true structure is not present in the input set of possible structures, or too many possible structures are given as input.

\section{ACKNOWLEDGMENTS}

The work is supported by the U.S. National Science Foundation under grant no. CCF-1422631 and CCF-1453563.

\section{REFERENCES}

[1] U. Andréasson, P. Edén, C. Peterson, C.-M. Högerkorp, M. Jerkeman, N. Andersen, M. Berglund, C. Sundström, R. Rosenquist, C. A. K. Borrebaeck, and S. Ek. 2010. Identification of uniquely expressed transcription factors in highly purified B-cell lymphoma samples. American fournal of Hematology 85, 6 (2010), 418-425.

[2] E. Avizienyte, A. Wyke, R. Jones, G. McLean, M. Westhoff, V. Brunton, and M. Frame. 2002. Src-induced de-regulation of E-cadherin in colon cancer cells requires integrin signalling. Nature Cell Biology 4, 8 (2002), 632-638.

[3] Y. Benjamini and Y. Hochberg. 1995. Controlling the false discovery rate: a practical and powerful approach to multiple testing. Fournal of the Royal Statistical Society. Series B (Methodological) (1995), 289-300.

[4] O. L. Caballero and Y.-T. Chen. 2012. Cancer/testis antigens: potential targets for immunotherapy. In Innate Immune Regulation and Cancer Immunotherapy. Springer, 347-369.

[5] K.-M. Chu, C. H. Cho, and V. Y. Shin. 2013. Nicotine and gastrointestinal disorders: its role in ulceration and cancer development. Current Pharmaceutical Design 19, 1 (2013), 5-10.

[6] D. Daria, N. Kirsten, A. Muranyi, M. Mulaw, S. Ihme, A. Kechter, M. Hollnagel, L. Bullinger, K. Döhner, H. Döhner, M. Feuring-Buske, and C. Buske. 2016. GPR56 contributes to the development of acute myeloid leukemia in mice. Leukemia 30 , 8 (2016), 1734.

[7] T. Do, E. Ucisik-Akkaya, C. Davis, B. Morrison, and M. Dorak. 2009. TP53 R72P and MDM2 SNP309 polymorphisms in modification of childhood acute lymphoblastic leukemia susceptibility. Cancer Genetics and Cytogenetics 195, 1 (2009), 31-36.

[8] R. Edgar, M. Domrachev, and A. E. Lash. 2002. Gene Expression Omnibus: NCBI gene expression and hybridization array data repository. Nucleic Acids Research 30, 1 (2002), 207-210.

[9] L. Fang, S. Lee, H. Choi, H. K. Kim, G. Jew, H. C. Kang, L. Chen, D. Jablons, and I.-J. Kim. 2014. Comprehensive genomic analyses of a metastatic colon cancer to the lung by whole exome sequencing and gene expression analysis. International fournal of Oncology 44, 1 (2014), 211-221.

[10] S. A. Farkas, V. Vymetalkova, L. Vodickova, P. Vodicka, and T. K. Nilsson. 2014. DNA methylation changes in genes frequently mutated in sporadic colorectal cancer and in the DNA repair and Wnt/ $\beta$-catenin signaling pathway genes. Epigenomics 6, 2 (2014), 179-191.

[11] W. Fiedler, H. Serve, H. Döhner, M. Schwittay, O. Ottmann, A.-M. O'Farrell, C. Bello, R. Allred, W. Manning, J. Cherrington, S. Louie, W. Hong, N. Brega, G. Massimini, P. Scigalla, W. Berdel, and D. Hossfeld. 2005. A phase 1 study of SU11248 in the treatment of patients with refractory or resistant acute myeloid leukemia (AML) or not amenable to conventional therapy for the disease. Blood 105, 3 (2005), 986-993.

[12] A. Foroughi pour and L. A. Dalton. 2014. Optimal Bayesian feature selection on high dimensional gene expression data. In Proceedings of the 2014 IEEE Global Conference on Signal and Information Processing (GlobalSIP). 1402-1405.

[13] A. Foroughi pour and L. A. Dalton. 2015. Optimal Bayesian feature filtering. In Proceedings of the 6th ACM Conference on Bioinformatics, Computational Biology and Health Informatics. 651-652.

[14] A. Foroughi pour and L. A. Dalton. 2016. Multiple sclerosis biomarker discovery via Bayesian feature selection. In Proceedings of the 7th ACM International Conference on Bioinformatics, Computational Biology, and Health Informatics. 540-541.

[15] A. Foroughi pour and L. A. Dalton. 2017. Robust feature selection for block covariance Bayesian models. In Proceedigns of 2017 IEEE International Conference on Acoustics, Speech and Signal Processing (ICASSP). 2696-2700.

[16] A. Foroughi pour and L. A. Dalton. 2018. Heuristic algorithms for feature selection under Bayesian models with block-diagonal covariance structure. $B M C$ Bioinformatics 19, 3 (2018), 70.

[17] S. Ganesh, C. F. M. Sier, G. Griffioen, H. J. M. Vloedgraven, A. de Boer, K. Welvaart, C. J. H. Van de Velde, J. H. J. M. Van Krieken, J. H. Verheijen, C. B. H. W. Lamers, 
and H. W. Verspaget. 1994. Prognostic relevance of plasminogen activators and their inhibitors in colorectal cancer. Cancer Research 54, 15 (1994), 4065-4071.

[18] M. Gjerstorff, M. Andersen, and H. Ditzel. 2015. Oncogenic cancer/testis antigens: prime candidates for immunotherapy. Oncotarget 6, 18 (2015), 15772-15787.

[19] T. R. Golub, G. F. Barker, M. Lovett, and D.Gary Gilliland. 1994. Fusion of PDGF receptor $\beta$ to a novel ets-like gene, tel, in chronic myelomonocytic leukemia with $\mathrm{t}(5 ; 12)$ chromosomal translocation. Cell 77, 2 (1994), $307-316$.

[20] M. González-González, C. Fontanillo, M. M. Abad, M. L. Gutiérrez, I. Mota, O. Bengoechea, Á. Santos-Briz, O. Blanco, E. Fonseca, J. Ciudad, , M. Fuentes, J. De Las Rivas, J. A. Alcazar, J. García, L. Muñoz-Bellvis, A. Orfao, and J. M. Sayagués. 2014. Identification of a characteristic copy number alteration profile by high-resolution single nucleotide polymorphism arrays associated with metastatic sporadic colorectal cancer. Cancer 120, 13 (2014), 1948-1959.

[21] A. L. Guzmán-Ortiz, G. Aparicio-Ozores, R. Valle-Rios, O. Medina-Contreras, G. Patiño-López, and H. Quezada. 2017. Proteomic changes in a childhood acute lymphoblastic leukemia cell line during the adaptation to vincristine. Boletín Médico Del Hospital Infantil de México (English Edition) 74, 3 (2017), 181-192.

[22] T. Haferlach, A. Kohlmann, L. Wieczorek, G. Basso, G. Te Kronnie, M.-C. Béné, J. De Vos, J. M. Hernández, W.-K. Hofmann, K. I. Mills, A. Gilkes, S. Chiaretti, S. A. Shurtleff, T. J. Kipps, L. Z. Rassenti, A. E. Yeoh, P. R. Papenhausen, W.-M. Liu, P. M. Williams, and R. Foá. 2010. Clinical utility of microarray-based gene expression profiling in the diagnosis and subclassification of leukemia: report from the international microarray innovations in leukemia study group. fournal of Clinical Oncology 28, 15 (2010), 2529-2537.

[23] J. Hua, W. D. Tembe, and E. R. Dougherty. 2009. Performance of feature-selection methods in the classification of high-dimension data. Pattern Recognition 42, 3 (2009), 409-424.

[24] Q. Huang, T. Whitington, P. Gao, J. F. Lindberg, Y. Yang, J. Sun, M.-R. Väisänen, R Szulkin, M. Annala, J. Yan, L. A. Egevad, K. Zhang, R. Lin, A. Jolma, M. Nykter, A. Manninen, F. Wiklund, M. H. Vaarala, T. Visakorpi, J. Xu, J. Taipale, and G.-H. Wei 2014. A prostate cancer susceptibility allele at 6q22 increases RFX6 expression by modulating HOXB13 chromatin binding. Nature Genetics 46, 2 (2014), 126-135.

[25] S. Ilyin, S. Belkowski, and C. Plata-Salamán. 2004. Biomarker discovery and validation: technologies and integrative approaches. Trends in Biotechnology 22, 8 (2004), 411-416.

[26] V. Jerome Marson, J. Mazieres, O. Groussard, O. Garcia, J. Berjaud, M. Dahan, P. Carles, and G. Daste. 2004. Expression of TTF-1 and cytokeratins in primary and secondary epithelial lung tumours: correlation with histological type and grade. Histopathology 45, 2 (2004), 125-134.

[27] D. Kanojia, M. Garg, S. Gupta, A. Gupta, and A. Suri. 2011. Sperm-associated antigen 9 is a novel biomarker for colorectal cancer and is involved in tumor growth and tumorigenicity. The American fournal of Pathology 178, 3 (2011), 1009-1020.

[28] J. Kendall, Q. Liu, A. Bakleh, A. Krasnitz, K. C. Q. Nguyen, B. Lakshmi, W. L. Gerald, S. Powers, and D. Mu. 2007. Oncogenic cooperation and coamplification of developmental transcription factor genes in lung cancer. Proceedings of the National Academy of Sciences 104, 42 (2007), 16663-16668.

[29] I. Kononenko, E. Šimec, and M. Robnik-Šikonja. 1997. Overcoming the myopia of inductive learning algorithms with RELIEFF. Applied Intelligence 7, 1 (1997) $39-55$.

[30] M. Konopleva, R. Contractor, T. Tsao, I. Samudio, P. P. Ruvolo, S. Kitada, X. Deng, D. Zhai, Y.-X. Shi, T. Sneed, M. Verhaegen, M. Soengas, V. R. Ruvolo, T. McQueen, W. D. Schober, J. C. Watt, T. Jiffar, X. Ling, F. C. Marini, D. Harris, M. Dietrich, Z Estrov, J. McCubrey, W. S. May, J. C. Reed, and M. Andreeff. 2006. Mechanisms of apoptosis sensitivity and resistance to the BH3 mimetic ABT-737 in acute myeloid leukemia. Cancer Cell 10, 5 (2006), 375 - 388.

[31] D. Lambert, I. Wood, A. Ellis, and S. Shirazi-Beechey. 2002. Molecular changes in the expression of human colonic nutrient transporters during the transition from normality to malignancy. British fournal of Cancer 86, 8 (2002), 1262-1269.

[32] T. Li, C. Zhang, and M. Ogihara. 2004. A comparative study of feature selection and multiclass classification methods for tissue classification based on gene expression. Bioinformatics 20, 15 (2004), 2429-2437.

[33] F. Liu, Y. Feng, Z. Li, C. Pan, Y. Su, R. Yang, L. Song, H. Duan, and N. Deng. 2014 Clinic-genomic association mining for colorectal cancer using publicly available datasets. BioMed Research International 2014 (2014). http://dx.doi.org/10.1155/ 2014/170289.

[34] H. Mi, X. Huang, A. Muruganujan, H. Tang, C. Mills, D. Kang, and P. D. Thomas. 2017. PANTHER version 11: expanded annotation data from gene ontology and Reactome pathways, and data analysis tool enhancements. Nucleic Acids Research 45, 1 (2017), $183-189$.

[35] H. Mi and P. Thomas. 2009. PANTHER pathway: an ontology-based pathway database coupled with data analysis tools. Humana Press, Totowa, NJ, 123-140

[36] Cancer Genome Atlas Research Network. 2013. Genomic and epigenomic landscapes of adult de novo acute myeloid leukemia. New England fournal of Medicine 368, 22 (2013), 2059-2074.

[37] J. E. Parker, G. J. Mufti, F. Rasool, A. Mijovic, S. Devereux, and A. Pagliuca 2000. The role of apoptosis, proliferation, and the Bcl-2-related proteins in the myelodysplastic syndromes and acute myeloid leukemia secondary to MDS. Blood 96, 12 (2000), 3932-3938.

[38] J. Peltier, J.-P. Roperch, S. Audebert, J.-P. Borg, and L. Camoin. 2016. Quantitative proteomic analysis exploring progression of colorectal cancer: modulation of the serpin family. Fournal of Proteomics 148 (2016), $139-148$.

[39] C. Peña, J. M. García, J. Silva, V. García, R. Rodríguez, I. Alonso, I. Millán, C. Salas, A. G. de Herreros, A. Muñoz, and F. Bonilla. 2005. E-cadherin and vitamin D receptor regulation by SNAIL and ZEB1 in colon cancer: clinicopathological correlations. Human Molecular Genetics 14, 22 (2005), 3361-3370.

[40] A. Foroughi pour and L. A. Dalton. 2016. Optimal Bayesian feature selection with missing data. In Proceedings of 2016 IEEE Global Conference on Signal and Information Processing (GlobalSIP). 35-39.

[41] A. Foroughi pour and L. A. Dalton. 2017. Integrating prior information with Bayesian feature selection. In Proceedings of the 8th ACM International Conference on Bioinformatics, Computational Biology, and Health Informatics. ACM, 610-610.

[42] A. Foroughi pour and L. A. Dalton. 2017. Multiclass Bayesian feature selection. In Proceedings of 2017 IEEE Global Conference on Signal and Information Processing (GlobalsIP). 725-729.

[43] A. Foroughi pour and L. A. Dalton. 2017. Optimal Bayesian feature filtering for single-nucleotide polymorphism data. In proceedings of 2017 IEEE International Conference on Bioinformatics and Biomedicine (BIBM). 2290-2292.

[44] T. Reya and H. Clevers. 2005. Wnt signalling in stem cells and cancer. Nature 434,7035 (2005), 843.

[45] G. Roffo and S. Melzi. 2016. Features Selection via eigenvector centrality. In Proceedings of New Frontiers in Mining Complex Patterns.

[46] C. Roth, H. Schmidberger, M. Lakomek, O. Witt, W. Wuttke, and H. Jarry. 2001. Reduction of $\gamma$-aminobutyric acid-ergic neurotransmission as a putative mechanism of radiation induced activation of the gonadotropin releasing-hormone-pulse generator leading to precocious puberty in female rats. Neuroscience Letters 297, 1 (2001), $45-48$

[47] S. Shin, T. Nagasaka, B. Jung, N. Matsubara, W. Kim, J. Carethers, C. Boland, and A. Goel. 2007. Epigenetic and genetic alterations in Netrin-1 receptors UNC5C and DCC in human colon cancer. Gastroenterology 133, 6 (2007), 1849-1857.

[48] C. Sima and E. R. Dougherty. 2006. What should be expected from feature selection in small-sample settings. Bioinformatics 22, 19 (2006), 2430-2436.

[49] C. Sima and E. R. Dougherty. 2008. The peaking phenomenon in the presence of feature-selection. Pattern Recognition Letters 29, 11 (2008), 1667-1674.

[50] A. Simpson, O. Caballero, A. Jungbluth, Y.-T. Chen, and L. Old. 2005. Cancer/testis antigens, gametogenesis and cancer. Nature Reviews Cancer 5, 8 (2005), 615-625.

[51] C. A. Sklar, L. L. Robison, M. E. Nesbit, H. N. Sather, A. T. Meadows, J. A. Ortega, T. H. Kim, and G. D. Hammond. 1990. Effects of radiation on testicular function in long-term survivors of childhood acute lymphoblastic leukemia: a report from the children cancer study group. Journal of Clinical Oncology 8, 12 (1990), 1981-1987.

[52] J. Smith, N. Deane, F. Wu, N. Merchant, B. Zhang, A. Jiang, P. Lu, J. Johnson, C. Schmidt, C. Bailey, S. Eschrich, C. Kis, S. Levy, M. Washington, M. Heslin, R. Coffey, T. Yeatman, Y. Shyr, and R. Beauchamp. 2010. Experimentally derived metastasis gene expression profile predicts recurrence and death in patients with colon cancer. Gastroenterology 138, 3 (2010), 958-968.

[53] L. Song, A. Smola, A. Gretton, K. Borgwardt, and J. Bedo. 2007. Supervised feature selection via dependence estimation. In Proceedings of the 24th International Conference on Machine Learning. 823-830.

[54] C. Tang, M. C. Willingham, J. C. Reed, T. Miyashita, S. Ray, V. Ponnathpur, Y. Huang, M. E. Mahoney, G. Bullock, and K. Bhalla. 1994. High levels of p26BCL2 oncoprotein retard taxol-induced apoptosis in human pre-B leukemia cells. Leukemia 8, 11 (1994), 1960-1969.

[55] M. Vincent and N. Hansen. 2014. Sparse group lasso and high dimensional multinomial classification. Computational Statistics \& Data Analysis 71 (2014), $771-786$.

[56] M. Z. Wojtukiewicz, L. R. Zacharski, V. A. Memoli, W. Kisiel, B. J. Kudryk, S. M. Rousseau, and D. C. Stump. 1989. Indirect activation of blood coagulation in colon cancer. Thrombosis and Haemostasis 62, 4 (1989), 1062-1066.

[57] H. P. S. Wong, L. Yu, E. K. Y. Lam, E. K. K. Tai, W. K. K. Wu, and C.-H. Cho. 2007. Nicotine promotes colon tumor growth and angiogenesis through $\beta$-adrenergic activation. Toxicological Sciences 97, 2 (2007), 279-287.

[58] Y. Xi, G. Nakajima, J. C. Schmitz, E. Chu, and J. Ju. 2006. Multi-level gene expression profiles affected by thymidylate synthase and 5 -fluorouracil in colon cancer. BMC Genomics 7, 1 (2006), 68.

[59] P. Yang, Z. Huo, H. Liao, and Q. Zhou. 2015. Cancer/testis antigens trigger epithelial-mesenchymal transition and genesis of cancer stem-like cells. Current Pharmaceutical Design 21, 10 (2015), 1292-1300.

[60] Y. N. Ye, E. S. L. Liu, V. Y. Shin, W. K. K. Wu, and C. H. Cho. 2004. The Modulating Role of Nuclear Factor- $\kappa \mathrm{B}$ in the Action of $\alpha 7$-Nicotinic Acetylcholine Receptor and Cross-Talk between 5-Lipoxygenase and Cyclooxygenase-2 in Colon Cancer Growth Induced by 4-(N-Methyl-N-nitrosamino)-1-(3-pyridyl)-1-butanone. Journal of Pharmacology and Experimental Therapeutics 311, 1 (2004), 123-130. 